\title{
La neurociencia aplicada en el ámbito educativo. El estudio de los neuromitos
}

Recibido: 28 de febrero de 2019 / Revisado: 27 de marzo de 2019

Aceptado: 29 de marzo de 2019 / Publicado: 21 de abril de 2019

MARTA MEDEL MONTERO

Centro Universitario Internacional de Madrid, España

JOSÉ ANTONIO CAMACHO CONDE

Facultad de Ciencias de la Educación, Universidad Internacional de la Rioja, España

joseantonio.camacho@unir.net

http://dx.doi.org/10.24310/IJNE2.1.2019.6559

\section{RESUMEN}

En el presente documento se realiza una revisión bibliográfica del campo de la Neurociencia, más concretamente de los diversos neuromitos que caracterizan a nuestra sociedad. Nuestro propósito es poder realizar un estudio y análisis del punto en el que se encuentran los mismos en las escuelas actuales, lo cual influye directamente en los procesos de enseñanza-aprendizaje de nuestro alumnado.

Con esto, se pretende conocer cómo la Neurociencia está siendo frenada por los mitos que creemos como verdaderos a pesar de que han sido refutados científicamente. Además, nos permite observar cómo y en qué magnitudes se encuentran arraigados estas ideas en los docentes.

Para responder al objetivo planteado en este estudio, se ha realizado una investigación-acción, en la que se ha recopilado el estado de diversos tópicos a través de un cuestionario cerrado realizado a maestros de un Centro de Educación Infantil y Primaria ubicado en la provincia de Guadalajara, contando con un total de 20 participantes que han mostrado sus conocimientos de

\section{ABSTRACT}

Neuroscience performed in education. The study of neuromite

In this document after doing a bibliographic review of the Neuroscience, specifically of the diverse neuromites that characterize our society. Our purpose is to be able to carry out a study and analysis of the point in which they are found in the current schools, which directly influences in the teaching-learning processes of our students.

With this study, we pretend to know how Neuroscience is being stopped by the myths that we believe to be true, but they have been refuted. In addition, it allows us to observe in what magnitudes they are rooted in teachers.

To meet the objectives of study, an empirical study has been carried out, in which a literature review to study the state of the Neuroscience. To complement it, has developed a questionnaire taken from different teachers from Infant and Primary School located in the province of Guadalajara with a total of 20 participants who have shown us their knowledge anonymously. This informa- 
manera anónima. Esta información se comparará con la realidad de los mitos para poner de manifiesto las ideas que frenan la utilización de la Neurociencia en Educación.

Tras su realización, los resultados obtenidos han sido bastantes sorprendentes a nivel general, mostrando el punto en el que nos encontramos, así como las ideas que tienen los docentes respecto a los neuromitos, lo cual frena en cierta medida la aplicación de la Neurociencia.

No obstante, queda demostrado en una pequeña muestra de la población que, aunque se traten de neuromitos actualmente refutados, se localiza la existencia de una gran multitud de ideas respecto a ellos, encontrándose una alta controversia y cierto desconocimiento del principal órgano del aprendizaje; el cerebro.

Palabras Clave: Educación, Neurociencia, Neuromitos, Cerebro, Enseñanza-aprendizaje. tion will be compared with the reality of the myths to highlight the ideas that hinder the use of Neuroscience in Education.

After this, the results have been quite surprising in general, showing the point where we are, as well as the ideas that teachers have about neuromites, which slows to the application of Neuroscience.

Although the neuromites are currently refuted, we find the existence of a great multitude of ideas about them. Also we see a high controversy and certain knowledge of the most important organ in learning, the brain.

Keywords: Education, Neuroscience, Neuromites, Brain, Teaching-learning.

\section{Introducción}

En las últimas décadas, la educación ha sido muy estudiada, sin embargo, ha sido en estos últimos años cuando se ha producido un mayor interés por el entendimiento de la Neurociencia y su aplicación en el proceso de enseñanza-aprendizaje, por lo que podemos encontrarnos ante lo que numerosos autores denominan la "pedagogía de futuro", debido a las revolucionarias aportaciones que la comportan.

Tanto ha ido creciendo la curiosidad por este ámbito que hoy estamos ante una "epidemia" cada vez mayor por la búsqueda de la dopamina en el aprendizaje, de manera especial por parte de los docentes, que buscan conocer todo aquello novedoso que ocurre en el campo de la educación para estar siempre actualizados y ofrecer una respuesta educativa adaptada a las necesidades de los alumnos y demandas de la sociedad.

Con este planteamiento, lo que se busca es concienciar sobre los grandes beneficios que aporta la Neuroeducación en el aprendizaje, potenciar los conocimientos de esta ciencia e ir erradicando los mitos arraigados en nuestra sociedad que la subyacen y la frenan de manera continuada. Con esto, se pretende la reflexión sobre la importancia del cerebro en el aprendizaje y de cómo podemos utilizarlo para favorecer la respuesta educativa a los alumnos, ya que como expone Mora (2018): 
“Todo lo que somos, lo que sentimos, lo que creemos, lo que pensamos, lo que hacemos en el mundo es producto del funcionamiento del cerebro. Por eso somos seres fundamentalmente emocionales y luego somos críticos y razonamos”.

Por consiguiente, la finalidad de este estudio radica en analizar las creencias y neuromitos que subyacen a la Neuroeducación, ya que pese a contar con innumerables opiniones y estudios a favor, se ha visto amenazada por diversas opiniones contrarias surgidas por estos "neuromitos" que la rodean, pasando desapercibida para muchos profesionales de la educación. No obstante, también se persigue el análisis de la acentuación de los neuromitos en docentes de Educación Infantil y Primaria, así como la comprobación del grado de conocimientos que tienen los docentes sobre el funcionamiento del cerebro.

La Neuroeducación; la última disciplina basada en el funcionamiento del cerebro en la adquisición de los aprendizajes a través de un enfoque integrador, procede de la combinación de conocimientos de la Neurociencia, la Psicología, la Pedagogía y la Sociología. Esta nueva concepción, surge con la finalidad de mejorar el proceso de enseñanza-aprendizaje a partir de las culturas acerca del funcionamiento del cerebro y de la adquisición de los conocimientos.

Por otro lado, el término neuromitos educativos hace referencia a las equívocas interpretaciones, ideas preconcebidas y falsas creencias relacionadas con el ámbito de la Neurociencia. Estos pensamientos erróneos, provienen generalmente de la cultura popular y publicaciones inexactas, que terminan por asentarse en nuestra red de conocimientos tomando gran posesión y fuerza en nuestras ideas. Por tanto, estas falsas premisas que carecen de fundamentación científica, están comenzando a derrumbarse gracias a las nuevas investigaciones, aunque todavía queda un largo camino por recorrer.

Existen multitud de neuromitos que vagan por nuestra sociedad, entre los cuales se destacan los siguientes:

\section{- Neuromito 1: Únicamente utilizamos el 10\% de nuestro cerebro}

Como todo neuromito, esta idea tiene su origen en el discurso social cotidiano, el cual se caracteriza por ser dogmático e irreflexivo, sin necesidad de validación científica. A esta situación, si se le añade el gran desconocido que era el cerebro hasta hace unos años, se construye la máxima de que sólo utilizamos esta ínfima parte del cerebro.

Tras varias investigaciones y experimentos, Pierre Flourens demuestra que las actividades sensoriales tenían una ubicación exacta y concreta en el cerebro mientras que las funciones superiores se encontraban repartidas en el cerebro, por lo que fue considerado como un órgano unitario. 
Estos experimentos, llevaron a que a finales del siglo XIX y principios del XX se aportara que en un momento determinado únicamente funcionaban el 10\% de las neuronas, ya que sólo se había podido mapear el 10\% del cerebro. Ante estas pesquisas, se fue modificando la información hacia el mito que escuchamos actualmente.

En la actualidad sabemos que las señales se transmiten a través del potencial de acción, el cual tiene un nivel gradual de eficacia en función de la distancia a recorrer, lo que provoca diversos niveles de potencia. Por ello, se dice que unas áreas están más encendidas que otras, es decir, que funcionan de una manera más rápida, lo que podría entenderse equívocamente como que utilizamos una pequeña parte del cerebro. Por tanto, sabemos que, aunque algunas partes se encuentran más activadas que otras, no se activan todas a la vez, sino que depende de los estímulos y de la información. No obstante, la suma de las diversas partes utilizadas demuestra a través de la neuroimagen que utilizamos la totalidad del cerebro.

\section{- Neuromito 2: Pasar más horas en la escuela significa más aprendizajes}

En la actualidad, se ha mostrado que no se aprende únicamente en la escuela en el horario estipulado, sino que los aprendizajes convergen de los diferentes entornos y contextos en los que se desarrolla el alumno, por lo que va más allá de las aulas. El aula debe ser concebida como una prolongación del hogar y de la sociedad, en donde se convive y se trabajan aspectos significativos y prácticos para la vida diaria.

No obstante, debido a ese ideal del aula como mejor sitio para la adquisición de conocimientos, en numerables ocasiones se piensa que cuantas más horas pasen los alumnos en la escuela, más aprenderán, pero la realidad es muy distinta, pues es preferible calidad antes que cantidad.

Ante esto, al ser un tema estático en la actualidad, en donde los horarios son fijos, se ha concluido que debido a que el tiempo atencional de los alumnos es reducido, no se aprende más por pasar más horas en la escuela de las estipuladas, ya que la curva de aprendizaje y de atención va disminuyendo según pasa el tiempo. Por ello Francisco Mora expone que el tiempo atencional de los alumnos no llega a los 50 minutos, por lo que la duración de las clases debe ser menor.

- Neuromito 3: La actividad física, el arte y el juego son elementos secundarios en la educación

Este mito resulta ser una gran realidad en las aulas, en donde el mismo marco legislativo en el que se dan las actuaciones de los docentes se encuentran con una ramificación de asignaturas en diferentes planos. En un primer lugar, siempre se destacan las áreas de Lengua Castellana y Literatura, así como Matemáticas, denominándose áreas troncales y con un mayor peso lectivo y temporal en el calendario. 
No obstante, hay asignaturas como las artes y el deporte, que cada vez son más relegadas a un segundo plano y con menos carga temporal, mostrándose como menos importantes para el desarrollo de los alumnos.

Diversos estudios realizados sobre el deporte y la educación han revelado que la actividad física proporciona serotonina, noradrenalina y dopamina entre otros neurotransmisores, lo cual potencia la atención, la motivación de los individuos y un aumento del hipocampo, esencial para la memoria explícita (recuerdos). No obstante, también se ha revelado gracias a Erickson que el ejercicio es beneficioso, ya que mejora la plasticidad sináptica, favorece la generación de nuevas neuronas en el hipocampo y aumenta la vascularidad cerebral.

Por otro lado, Hillman en 2009 demostró que los alumnos tenían un rendimiento académico superior tras la realización de deporte, mejorando la atención ejecutiva y el autocontrol.

Respecto a la educación artística, gracias a esta área los alumnos pueden conocer el mundo que les rodea de una manera más vivencial, mostrando grandes beneficios a nivel cognitivo como en el resto de áreas. Esto se debe a que las artes nos permiten desarrollar una serie de competencias que son inviables de lograr de otro modo. Además de mejorar las habilidades ya expuestas, se favorece el pensamiento abstracto, la creatividad e imaginación y un pensamiento más profundo del entorno, logrando así aprendizajes más cercanos a la realidad.

\section{- Neuromito 4: Para lograr un aprendizaje es necesario salir de nuestra zona de confort}

Para lograr aprendizajes no es necesario salir de la zona de confort, aunque es algo altamente recomendable si queremos ciudadanos competentes del siglo en el que nos encontramos. Sin embargo, la Neurociencia nos indica que el mejor modo de aprender es a través del gozo intelectual (Wagensberg: 2007), que es aquel que acontece ante una nueva comprensión y aprendizaje.

Además del gozo, se requiere de emociones como el asombro, ya que es el mecanismo a través del cual un individuo conoce algo desconocido para él anteriormente, lo que vendría siendo el propio aprendizaje. Por lo tanto, a través de la indagación y del descubrimiento, salgamos o no de nuestra zona de confort se produce un aprendizaje.

\section{- Neuromito 5: Escuchar obras de Mozart hace que seamos más inteligentes}

Es cierto que escuchar música tiene diversos efectos en el cerebro y en el cuerpo de los seres humanos, de ahí que sea una parte esencial de nuestra vida y recurramos a ella en diferentes situaciones a lo largo de nuestra vida. 
La educación musical conlleva multitud de beneficios a nivel cognitivo, por lo que es esencial tratarla de manera adecuada en el currículo, especialmente en las primeras etapas educativas, no obstante, no hay ninguna evidencia ni prueba científica que verifique esta falsa premisa

Varios estudios realizados por Zimmerman demuestran que procesar algo tan complejo como es la música clásica, pese a parecer que puede gustar en esas edades tan tempranas, no tiene en cuenta el desarrollo neuronal del oyente, por lo que puede provocar efectos perjudiciales como la sobreestimulación, déficit de atención y escasa calidad de sueño en el futuro.

\section{- Neuromito 6: Con trabajo y seriedad se facilitan los aprendizajes}

Gracias a la Neurociencia, se ha revelado que el cerebro humano posee una serie de sistemas que favorecen la motivación, la atención y el aprendizaje eficaz, lo que aporta nuevos paradigmas educativos, dejando atrás la idea de que la seriedad y el control favorecen los aprendizajes, ya que como indica Francisco Mora "Sólo se puede enseñar a través de la alegría".

Posteriormente se descubrieron los beneficios placenteros a nivel cerebral que posee la segregación de dopamina, ejerciendo la labor de reforzador ante estímulos agradables. Por ello, se ha investigado su gran capacidad en lo referente a la regulación de la motivación, lo que potencia el foco hacia nuevos aprendizajes, ya que la dopamina, junto con otros factores, proporciona ayuda en la toma de decisiones. Por tanto, si la dopamina que se segrega ante momentos placenteros provoca mejoras en los aprendizajes, queda refutada.

\section{- Neuromito 7: El proceso de enseñanza-aprendizaje es guiado por el hemisferio cere- bral predominante}

Ante esta información, la Neurociencia ha demostrado que hay ciertas áreas cerebrales que tienen unas funciones concretas, y que puede haber la existencia de la predominancia de hemisferios en relación con algunas actividades. No obstante, debido a que el cerebro posee un funcionamiento altamente complejo, generalmente se requiere de la integración de las diversas áreas para un correcto funcionamiento.

Como se viene diciendo, se encuentra demostrado que los dos hemisferios no se encuentras aislados entre sí, sino que demuestran una gran y continua interconexión a través del cuerpo calloso. Sin embargo, se pasa a aclarar algunas cuestiones que han seguido vigentes hasta la actualidad:

- El hemisferio izquierdo no es lingüístico, pues al utilizar las habilidades y estrategias propias de la comunicación y la lengua, intervienen diversas áreas cerebrales que se encuentran en hemisferios diferentes, pero con una alta conexión neuronal. 
- La creatividad no es inherente al hemisferio derecho, pues este proceso de crear conlleva la utilización de ambos hemisferios.

- Del mismo modo, las matemáticas no se relacionan directamente con el hemisferio izquierdo, ya que se ha identificado mediante las nuevas tecnologías que la utilización de los números activa diversas áreas cerebrales.

- La música es otro claro ejemplo de interconectividad cerebral, pues no pertenece únicamente al hemisferio derecho como anteriormente se pensaba, ya que requiere de procesos de percepción melódica y rítmica que se encuentran en el hemisferio izquierdo.

\section{Material y método}

Como indican y definen Cohen y Manion (1994:42) el modelo de investigación-acción es un: "Procedimiento centrado en su objeto y pensado para tratar un problema concreto localizado en una situación inmediata". Este proceso de investigación-acción que se ha llevado a cabo precisa valerse de una técnica de recogida de datos para extraer y analizar la información necesaria para este estudio.

La técnica utilizada en esta investigación ha sido el análisis de un cuestionario con 3 ítems de evaluación ( $\mathrm{Si}$, No, No sé), realizada con una muestra de 20 participantes, todos ellos hombres y mujeres docentes de un Centro de Educación Infantil y Primaria de la provincia de Guadalajara.

A través de ese cuestionario, se analizará el estado actual de los neuromitos así como el conocimiento de los docentes sobre el funcionamiento del cerebro en la adquisición de aprendizajes.

Se ha seleccionado esta técnica debido a que, tras una toma de decisiones, esta opción fue considerada la más apropiada y eficaz para obtener información real de las ideas y conocimientos de los docentes, así como del proceso de enseñanza-aprendizaje en el que se desarrollan los diversos niños alcarreños, ya que al ser de manera anónima se garantiza una mayor veracidad de las respuestas.

\section{Resultados}

A partir de las respuestas dadas por parte de los participantes se han obtenido los siguientes resultados:

- Neuromito 1: Únicamente utilizamos el 10\% de nuestro cerebro. Como se puede observar, el $47 \%$ niega este mito. No obstante, no podemos olvidarnos del $29 \%$ que sigue creyendo esta teoría y del $24 \%$ que desconocía tanto si era cierta como incierta (ver figura 1). 


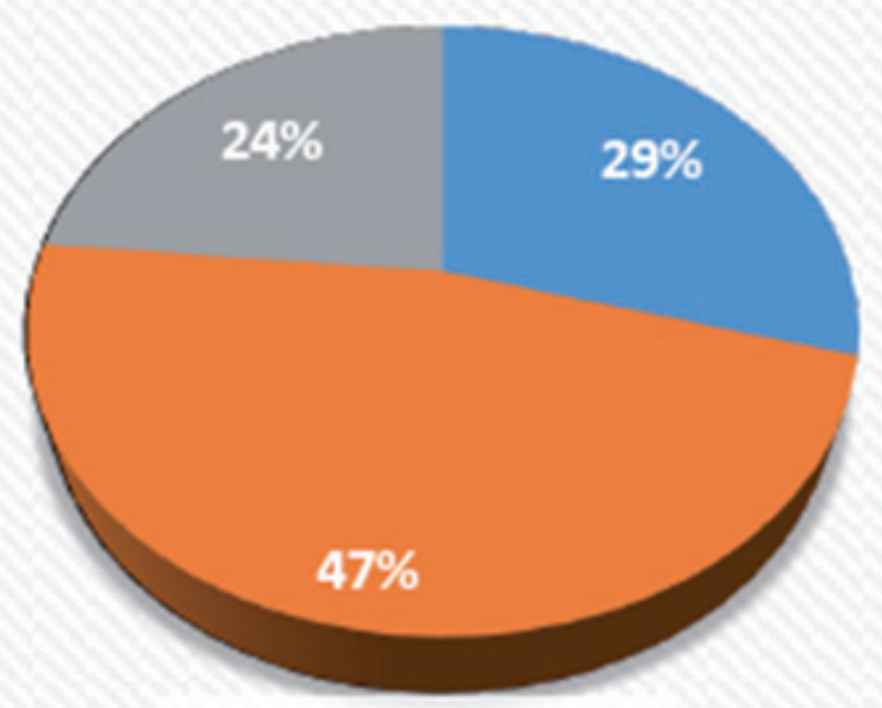

$\square \mathrm{SI} \square \mathrm{NO} \square \mathrm{NOSE}$

Figura 1. Gráfica sobre la utilización del 10\% del cerebro

- Neuromito 2: Pasar más horas en la escuela significa más aprendizajes. Prácticamente, la totalidad de los docentes tienen refutado este mito dentro de sus ideales. No obstante, hay un ligero $12 \%$ de los encuestados que desconocía si esto era cierto o incierto (ver figura 2).

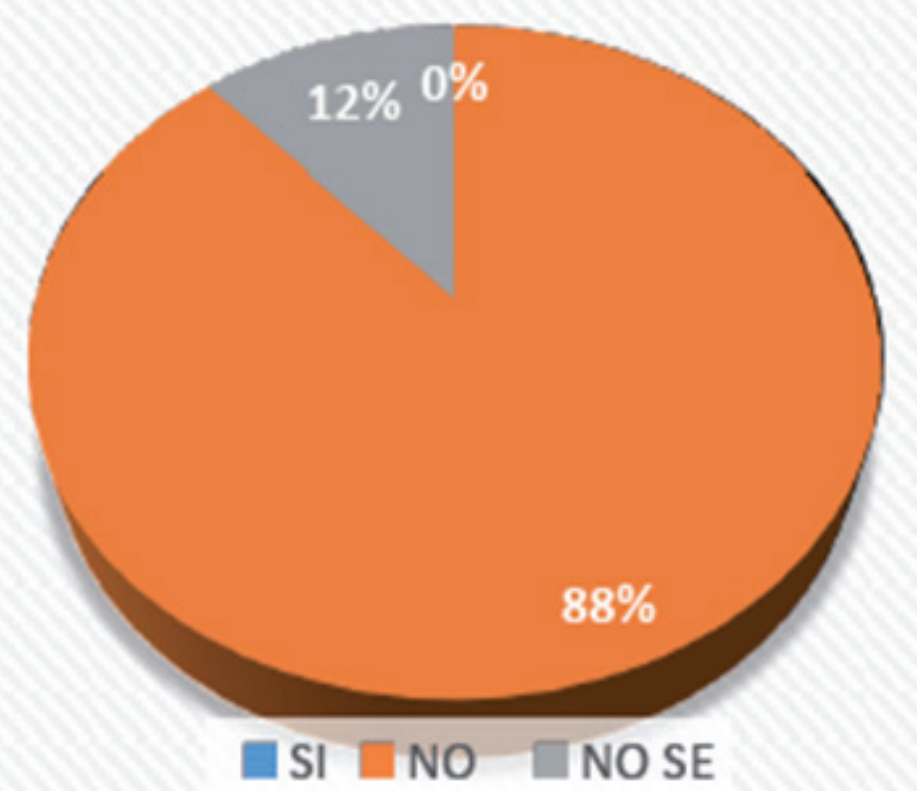

Figura 2. Gráfica sobre la relación horas en la escuela y aprendizaje

- Neuromito 3: La actividad física, el arte y el juego son elementos secundarios en la educación. Prácticamente todos los docentes, más concretamente un 94\% como se muestra en la figura, opina 
lo contrario a este mito; viendo la importancia que supone estas materias en el desarrollo integral de los alumnos (ver figura 3).

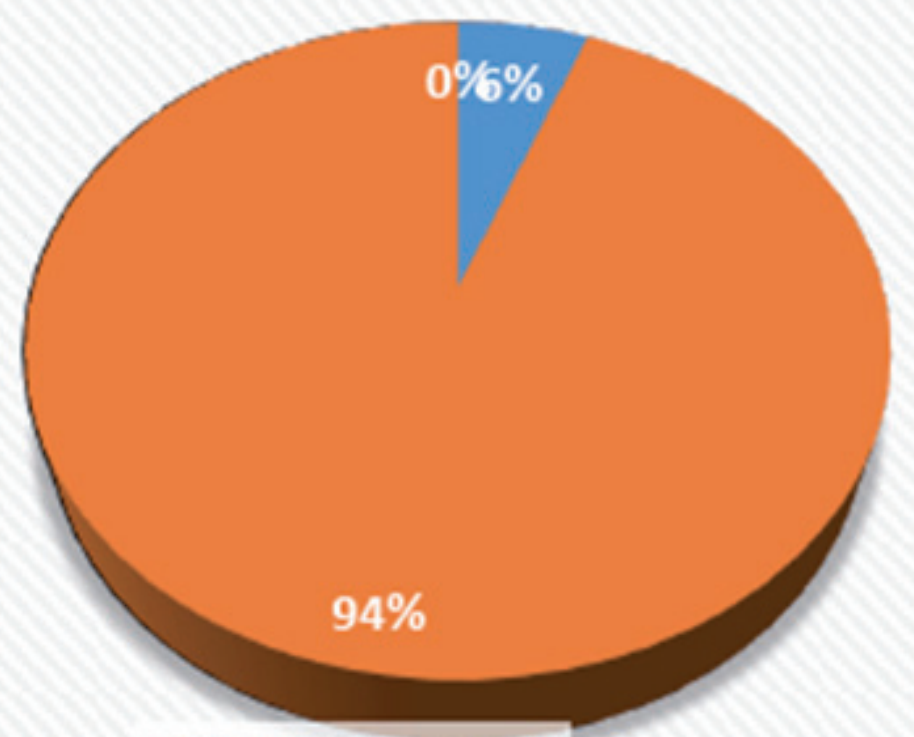

SI $1 \mathrm{NO} \square \mathrm{NOSE}$

Figura 3. Gráfica sobre la relación artes y deporte y su ocupación de un segundo plano en educación

- Neuromito 4: Para lograr un aprendizaje es necesario salir de nuestra zona de confort. Directamente proporcional a la teoría expuesta, como se puede observar en la figura, un $76 \%$ de los encuestados no cree que para lograr un aprendizaje sea necesario salir de la zona de confort (ver figura 4).

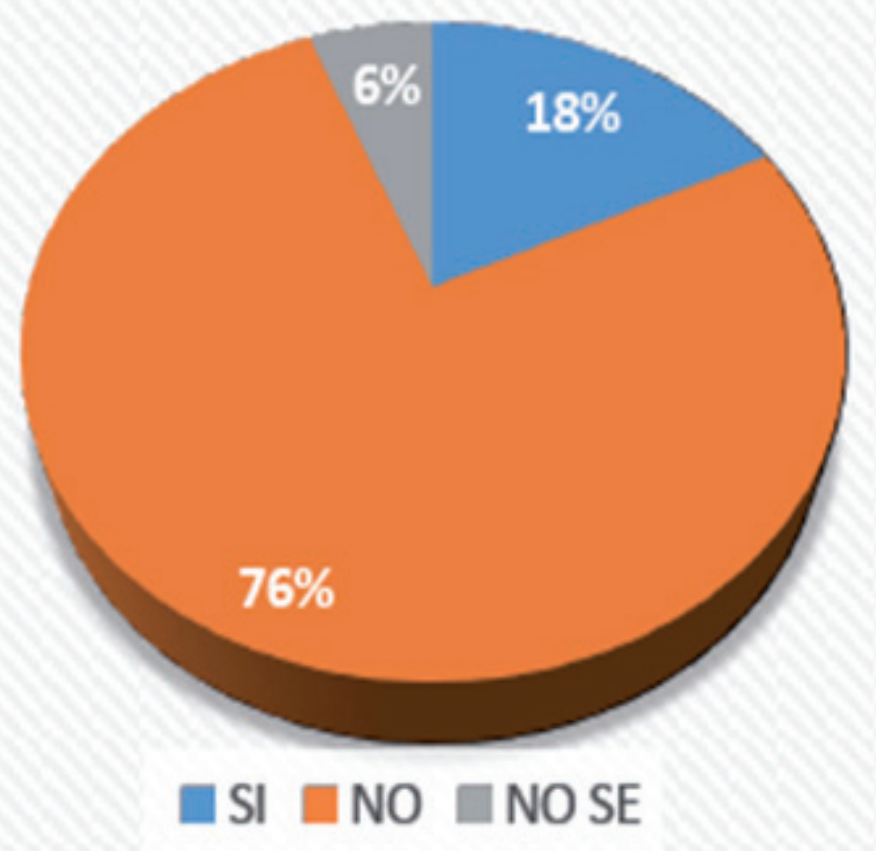

Figura 4. Gráfica sobre la relación aprendizaje y zona de confort 
- Neuromito 5: Escuchar obras de Mozart hace que seamos más inteligentes. Un 29\% de docentes, ha respondido con aseidad que Mozart influye la inteligencia de las personas, frente a un $47 \%$ que desmiente esta teoría. (ver figura 5).

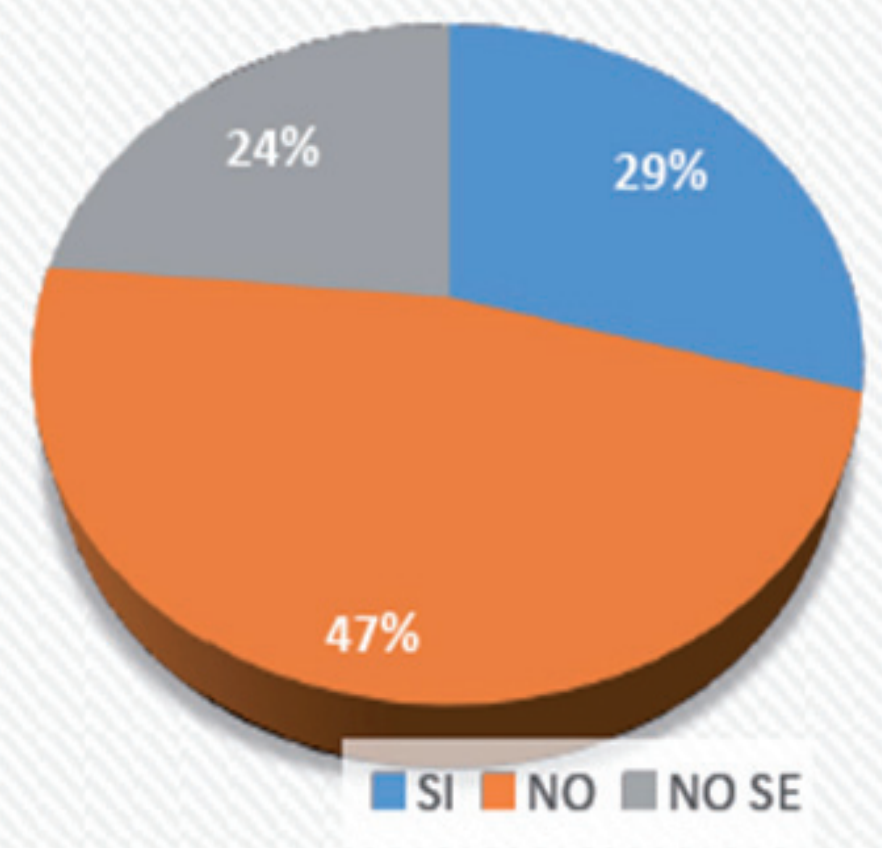

Figura 5. Gráfica sobre escuchar Mozart nos hace más inteligentes

- Neuromito 6: Con trabajo y seriedad se facilitan los aprendizajes. Como se puede observar, los resultados obtenidos muestran un claro empate entre los partidarios de la seriedad de la escuela con un $41 \%$ y, los partidarios de un ambiente más permisivo y democrático con un 53\% (ver figura 6).

- Neuromito 7: El proceso de enseñanza-aprendizaje es guiado por el hemisferio cerebral predominante. Pese a encontrase científicamente probado que ambos hemisferios cerebrales fluctúan información de manera continua en las diversas actividades, un 23\% de los encuestados lo afirman y un $24 \%$ no identifican esta relación. No obstante, un 53\% niegan esta situación porque conciben el cerebro como un ser unido e interconectado entre sí (ver figura 7).

\section{Discusión}

De todos los mitos comentados, los resultados obtenidos nos muestran cómo a nivel general siguen la gran mayoría de los neuromitos ligeramente vigentes en nuestra sociedad, en un mayor o menor porcentaje, pese a los estudios y experimentos que los refutan. Estos neuromitos que quedan por resolver en la actualidad se deben a su gran controversia y desconocimiento del principal órgano del aprendizaje; el cerebro. 


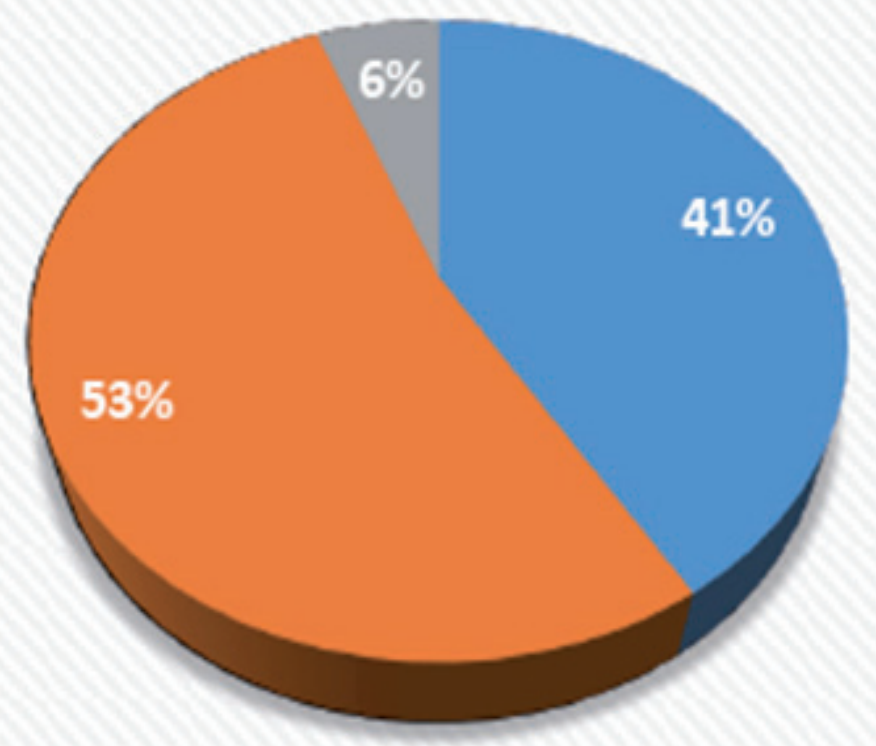

SI NO NOSE

Figura 6. Gráfica sobre el trabajo y la seriedad facilitan los aprendizajes

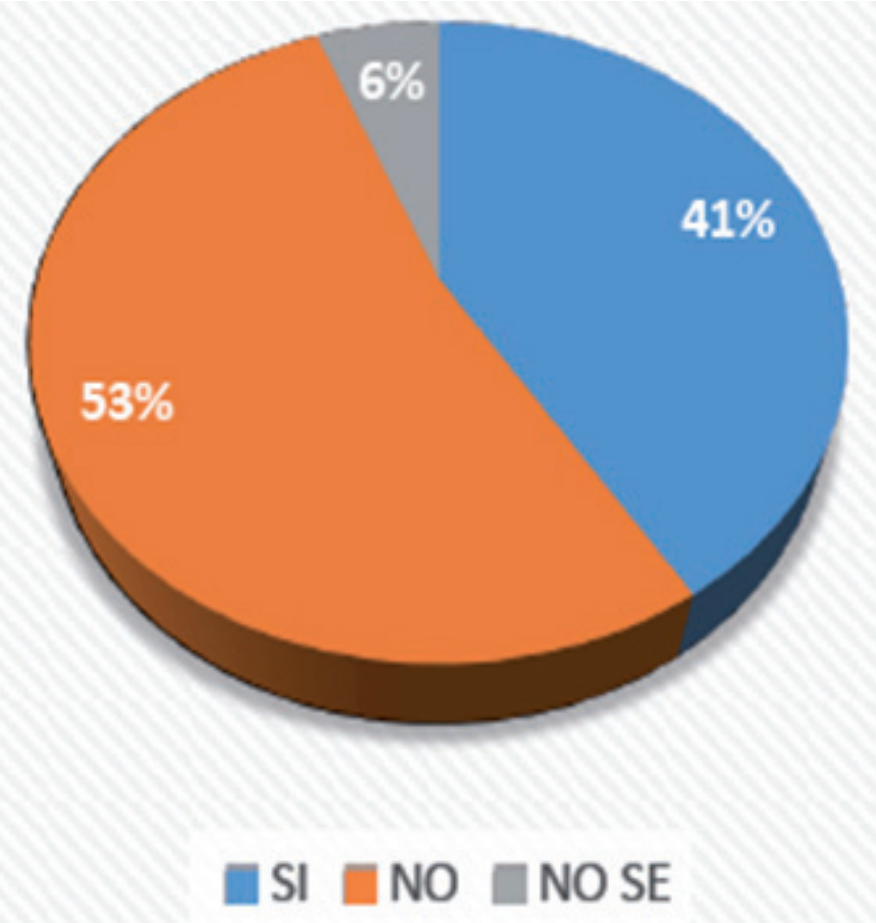

Figura 7. Gráfica sobre el hemisferio cerebral predominante 
No obstante, algunas cuestiones como la relación entre el tiempo en la escuela y los aprendizajes, así como la importancia de las artes y el deporte en educación, se perciben ciertos indicios de cambios en un mayor volumen de la muestra estudiada, ya que lo han negado un $88 \%$ y un $94 \%$ respectivamente, acercándose a las bases de la Neurociencia, aunque todavía, estos conocimientos no han llegado a todo el sector educativo.

Es necesario resaltar la multiplicidad de opiniones respecto al porcentaje de utilización del cerebro, en donde se muestra una clara controversia de ideas entre los docentes encuestados. Se aprecia que un $47 \%$ lo refuta mientras que la otra mitad de la muestra bien duda de su utilización o lo afirma. Esto nos muestra la necesidad de formación que se requiere en este ámbito, ya que la mitad de los maestros encuestados poseen ciertas dudas sobre cómo funciona el cerebro en su totalidad y su utilización, aunque como se viene comentando, se observa un amplio camino hacia el cambio.

Esta misma situación de controversia, ocurre con otro gran neuromito por excelencia, referido al aumento de nuestra inteligencia al escuchar a Mozart. Pese a que Abott en 2007, en su estudio sobre el "Efecto Mozart", desveló que no detectó correlaciones entre la escucha de la obra de Mozart y la mejora de las habilidades cognitivas, demostró que en los escasos casos que sí se detectaron, no pasaban de la media hora, por lo que no supone ninguna mejoría significativa. A esto se le suma el descubrimiento de que esta misma situación se daba con igual probabilidad con otros tipos de música diferentes a Mozart así como otras experiencias agradables para el sujeto, por lo que se concluyó que escuchar a Mozart no aumenta nuestro Coeficiente Intelectual.

Pese al camino que queda por recorrer en este ámbito, resulta llamativo que, en todas ocasiones, el porcentaje mayor sea el reticente del mito encuestado, mostrando esos indicios de cambio que se comentaban con anterioridad. Pese a las controversias obtenidas en algunas cuestiones, el mayor porcentaje de respuestas siempre son las refutantes del mito propuesto, demostrando en todo momento, a nivel general, un elevado número de los encuestados es conocedor del funcionamiento del cerebro.

Con una mayor información y formación no sólo del sector educativo, sino en donde toda la población sea partícipe, estos mitos se irán desenraizando de nuestros ideales, lo que además de otorgarnos un mayor conocimiento sobre el cerebro y su funcionamiento, promoverá un proceso de enseñanza-aprendizaje más individualizado a nuestro alumnado.

\section{Conclusiones}

La neuroeducación ha aparecido para quedarse en todo proceso de enseñanza-aprendizaje, ya que la sociedad educativa no puede mantenerse al margen del funcionamiento del cerebro, un órgano esencial 
para el aprendizaje a la par que complejo, debido a todas las funciones cognitivas que realiza. Todavía queda un largo camino por recorrer para que la Neurociencia ahonde más en el sistema educativo, ya que aún la comunidad educativa, aunque cada vez se encuentra más formada en este ámbito, todavía quedan restos de ideas preconcebidas sobre el cerebro.

Resulta interesante que pese a ser el órgano principal en donde convergen todos los aprendizajes, tanto formales como no formales, haya todavía controversias con su funcionamiento. Puesto que de ser al contrario podrían beneficiarse de la multiplicidad de opciones que nos permite adaptar las actividades a todos los alumnos en función de sus necesidades, ya que, aunque requieran de métodos distintos, siempre hay acciones y procesos que benefician a todos los cerebros de una manera más general gracias a la formación docente, lo cual es un camino largo y continuo de aprendizaje. Ya que como dice Stanislas Dehaene:

“¿Por qué debe esperarse que cada maestro redescubra de inmediato y por su cuenta -por tanteos, sin sacar provecho de los estudios científicos existentes aquello que a los investigadores les ha tomado décadas comprender?”.

\section{Limitaciones del estudio}

Para finalizar, destacar que este estudio cuenta con ciertas limitaciones que impiden de una mayor generalización de los resultados. Entre ellas nos encontramos con el tamaño de la muestra.

El estudio se ha realizado con la participación de 20 personas, por lo que, aunque los datos son extrapolables, no pueden ser universales, ya que pueden variar si se contase con una muestra más representativa de la sociedad. Asimismo, otro factor a tener en cuenta es la cantidad de datos obtenidos, los cuales son muy concretos al realizarse cuestionarios cerrados en vez de abiertos.

Al utilizar esta metodología no se han obtenido datos exhaustivos y más argumentados en la investigación. Esto, en retrospectiva, podría haber ayudado a conocer las inquietudes y conocimientos de los docentes que no han refutado los mitos y/ o dudaban de su veracidad.

\section{Conflicto de intereses}

Los autores no han recibido financiación para este estudio y no manifiestan ningún conflicto de intereses en el proceso de la redacción, revisión y publicación de este artículo. 


\section{Referencias}

Abbott, A. (2007). Mozart doesn't make you clever. Nature News. Recuperado el 18/05/2018 de: https://www.nature.com/news/2007/070409/full/news070409-13.html

Bajo, T., Fernández, A., Ruiz M. \& Gómez-Ariza, C.J. (2016). Memoria: estructura y funciones. Madrid: Alianza Editorial.

Barceló, F. (2004) ¿Neurociencia cognitiva o moderna neurociencia? Cognitiva. Vol. 16, № 2 , 2004, págs. 163-168.

Berwick, R. C., Friederici, A. D., Chomsky, N., \& Bolhuis, J. J. (2013). Evolution, brain, and the nature of language. Trends in cognitive sciences, 17(2), 89-98.

Bisquerra, R. ¿Qué son las emociones? Grupo de Investigación en Orientación Psicopedagógica (GROP). Recuperado el 15/03/2018 de: http://www.rafaelbisquerra.com/es/biografia/publicaciones/articulos/101-educacion-emocional-competencias-basicas-para-vida/208-concepto-emocion.html

Castaño, J. (2003). Bases neurobiológicas del lenguaje y sus alteraciones. Revista de Neurología, 36(8), págs. 781-785.

Cohen, L. y Manion, L. (1994). Research Methods in Education. Londres: Routledge. Recuperado el 18/03/2018 de: http://knowledgeportal.pakteachers.org/sites/knowledgeportal.pakteachers.org/files/resources/RESEARCH\%20METHOD\%20COHEN\%20ok.pdf

Dennison, P.E., \& Dennison, G.E. (2012). Brain Gym. Movimientos para mejorar en tu vida. Barcelona: Vida kinesiológica.

Forés, A., Gamo, J.R., Guillén, J.C, Hernández, T., Ligoiz, M., Pardo, F. \& Trinidad, C. (2015). Neuromitos en Educación. El aprendizaje desde la neurociencia. Barcelona: Plataforma editorial.

Gazzaniga, M. S. (2012). ¿Quién manda aquí? El libre albedrío y la ciencia del cerebro. Barcelona: Paidós.

Guillén, J.C. (2012). Neuroeducación: Estrategias basadas en el funcionamiento del cerebro. Escuela con cerebro. Recuperado el 15/05/2019 de: https://escuelaconcerebro.wordpress. com/2012/12/27/neuroeducacion-estrategias-basadas-en-el-funcionamiento-del-cerebro/ 
Guillén, J.C. (2017). Neuroeducación en el aula. De la teoría a la práctica. CreateSpace.

Guzmán, G. Áreas del cerebro especializadas en el lenguaje: su ubicación y funciones. Psicología y mente. Recuperado el 10/05/2018 de: https://psicologiaymente.net/neurociencias/areasdel-cerebro-lenguaje

Hillman, C., et al (2009). The effect of acute treadmill walking on cognitive control academic achievement in preadolescent children. Neuroscience, (159), págs. 1044-1054.

Lavilla Cerdán, L. (2011). La memoria en el proceso de enseñanza/ aprendizaje. Pedagogía Magna. No 11, págs.. 311-319. Recuperado el 15/05/2018 de: https://dialnet.unirioja.es/servlet/ articulo? codigo $=3629232$

Mora, F. (2000). El cerebro siente. Barcelona: Ariel.

Mora, F. (2007). Neurocultura. Madrid: Alianza Editorial.

Mora, F. (2009). Cómo funciona el cerebro. Madrid: Alianza Editorial.

Mora, F. (2016). El cerebro juega con las ideas. Madrid: Alianza Editorial.

OCDE. (2007). Understanding the brain: the birth of a learning science. París: OCED.

Ruiz, J.M. (1994). La memoria humana: Función y estructura. Madrid: Alianza Editorial.

Stickgold, R., James, L., \& Hobson, J. A. (2000). Visual discrimination learning requires sleep after training. Nature neuroscience, 3(12), 1237. Recuperado el 24/05/2018 de https://www. nature.com/articles/nn1200_1237.pdf

Wagensberg, J. (2007). El gozo intelectual. Barcelona: Tusquets. 